\title{
Immunohistochemical expression of VEGF-A and its ligands in non-neoplastic lesions of the breast sampling-assisted by dynamic angiothermography
}

\author{
ADHEMAR LONGATTO FILHO ${ }^{1}$, SANDRA M. COSTA $^{1}$, FERNANDA MILANEZI ${ }^{1,2}$, DANIELE MONTRUCCOLI ${ }^{3}$, \\ GIAN CARLO MONTRUCCOLI ${ }^{4}$, FáTIMA BALTAZAR ${ }^{1}$ and FERNANDO C. SCHMITT ${ }^{2,5}$

\begin{abstract}
${ }^{1}$ Life and Health Sciences Research Institute, School of Health Sciences, University of Minho, Campus of Gualtar, Braga;
${ }^{2}$ Institute of Molecular Pathology and Immunology of University of Porto (IPATIMUP), Porto, Portugal; ${ }^{3}$ Department of

Surgery Pietro Valdoni, University of Rome La Sapienza, Rome; ${ }^{4}$ Department of Obstetrics and Gynecology,

Toniolo Private Clinic, Bologna, Italy; ${ }^{5}$ Medical Faculty of University of Porto, Porto, Portugal
\end{abstract}

Received May 8, 2007; Accepted June 27, 2007

\begin{abstract}
The aim of this study was to investigate the expression of angiogenic markers, vascular endothelial growth factor A (VEGF-A) ligand and its receptors, VEGFR1 and -2 , in a series of biopsy-proven non-neoplastic lesions of the breast detected by dynamic angiothermography. We have also studied the vascular density demonstrated by CD31 immunoreactivity, in order to assess the potential of the imaging method to recognize lesions with an enhaced vascular network of clinical importance in routine breast examination. The lesions were classified as non-proliferative, proliferative without atypia and proliferative with atypia. VEGF was diffusely expressed in the epithelial cells of proliferative lesions in almost all cases. Similarly, VEGFR-1 and -2 also exhibited epithelial positive reactions in the majority of cases. VEGF-A and its receptors were also present in blood vessels. CD31 showed an increase in vascular proliferation at the periphery of proliferative epithelial lesions, but not in non-proliferative lesions. Our results, showing marked expression of VEGF by the epithelial proliferative lesions and neoangiogenesis at their periphery, confirm that these lesions can be detected by dynamic angiothermography.
\end{abstract}

\section{Introduction}

Breast carcinoma is one of the most prevalent cancers in women worlwide, accounting for thousands of cancer-related

Correspondence to: Dr Fernando C. Schmitt, Institute of Molecular Pathology and Immunology of University of Porto (IPATIMUP), Rua Roberto Frias s/n, 4200 Porto, Portugal

E-mail: fschmitt@ipatimup.pt

Key words: VEGF, VEGFR-1, VEGFR-2, CD31, breast cancer, angiothermography, dynamic angiothermography, thermography, breast disease deaths annualy, despite significant advances in early detection and treatment (1). The majority of breast carcinoma cases are pathologically diagnosed as invasive or infiltrating where they have spread beyond the duct or lobule of origin, invading the surrounding tissue of the breast (2). In the last few years, advances in cancer prevention and therapeutics have led to significant declines in the rates of cancer incidence and mortality and increases in life expectancy and survival for many cancer patients (2). The prognosis of early breast cancer is generally favorable. Particularly, ductal carcinoma in situ has been regarded as a non-life-threatening disease (3).

A plethora of growth factors and their associated receptors, steroid hormones and receptors, and proteinases are involved in regulation of breast tumor cells. Among growth factors, vascular endothelial growth factor (VEGF) is believed to be one of the most important molecular players in breast carcinoma, because higher levels of cytosolic VEGF-A are a powerful independent prognostic factor both in node-negative and node-positive breast cancers (4).

VEGF-A exerts its effects predominantly through two receptor tyrosine kinases, VEGFR-1 and VEGFR-2. While functional VEGF-A receptors are principally expressed on endothelial cells, it is now well established that VEGFR-1 is expressed on other cell types, including hematopoietic cells, monocytes and smooth muscle cells (5). Increased VEGFR-1 expression in breast carcinomas is accepted as a significant prognostic indicator of poor outcome, high risk of metastasis and relapse risk (6,7). Interestingly, VEGFR-2 immunohistochemical positivity on the tumor surface did not correlate with survival or with metastasis and relapse risk (8). However, VEGFR-2 is the most important receptor of VEGF in physiological and pathological conditions $(9,10)$.

New methodological options have been investigated in order to optimize diagnosis and evaluate risk for breast cancer. Novel thermography applications are also being used for cancer detection. The thermal imaging technology is based on the principle that heat associates to abnormal endothelial cell proliferation (11). Thermography is an easily reproducible method and has conveniently low costs when 
compared to mammography. Additionally, mammography interpretation is largely dependent on the radiologist. Thermography examination involves no radiation risk as it only captures the infrared radiation from the skin and is also totally painless. However, thermography is only a test of physiology, whereas mammography is presently referred to as the 'gold-standard' in breast cancer detection (12). Thermography has been comprehensively used as a cancer detection apparatus, but has not been accepted as well as mammography because of the incidence of high false positive cases. Furthermore, thermography only indicates outward abnormalities whereas mammography provides anatomical information of the abnormality. Interestingly, despite this, patients with abnormal false positive thermography results are at a higher risk of contracting breast cancer (13).

In spite of some criticism concerning thermography, we hypothesized that the reason for some imprecise thermal evaluations was that the images were an aggregate sum of the numerous biases consequent to heat produced by the underlying normal breast tissue, by neoplastic cell metabolism and by the blood supply itself (14). Accordingly, by excluding blood-supply heat, and verifying angiogenesisderived variations, we developed a new method that was able to work faster, with better spatial resolution and more sensitivity in order to provide a functional and qualitative, rather than quantitative, reading and recording of the images in vivo. The method was appropriately tested in collaboration with the University of Bologna's Department of Physics (Health Physics Division); the results always matched mammography diagnosis, and have been increasingly satisfactory (15).

The goal of this study was to investigate if non-neoplastic lesions with vascular proliferation were also eligible to be detected by dynamic angiothermography. We also investigated the expression of molecular angiogenic players such as VEGFA and its receptors VEGFR-1 and VEGFR-2 in epithelial ductal cells and surrounding blood vessels.

\section{Materials and methods}

Patients and tumour samples. Biopsy-proven non-neoplastic lesions of the breast detected by dynamic angiothermography were obtained from 39 women, from the pathology files of the Toniolo Private Clinic, Bologna, Italy. All histopathological diagnoses were revised by two of the authors (FM and FS) and categorized according to the WHO classification (16). For statistical purposes these lesions were classified as nonproliferative $(n=6)$, proliferative without atypia $(n=24)$ and proliferative with atypia $(n=10)$.

Most of the non-malignant lesions of the breast were associated with more than one type of alteration. Briefly, the main categorization was as follows: i) non-proliferative lesions, encompassing 6 cases of cysts; ii) proliferative lesions without atypia comprising 13 cases of adenoses combined with usual ductal hyperplasia (UDH), 3 cases with predominantly UDH characteristics, 7 cases of columnar alteration with prominent apical snouts and secretions (CAPSS), four of them associated to adenoses, and 1 case of pseudoangiomatous stromal hyperplasia (PASH) associated with UDH; iii) the remaining ten cases exhibited miscellaneous features of benign lesions, all with foci of atypical hyperplasia (AH).
VEGF-A, VEGFR-1, VEGFR-2 and CD31 immunohistochemical procedure. Immunohistochemistry was carried out with the streptavidin-biotin-peroxidase complex technique [Ultravision detection system anti-polyvalent, HRP (Lab Vision Corporation, Fremont, CA, USA) for VEGF-A and CD31; DakoCytomation LSAB2 ${ }^{\circledR}$ system-HRP (DakoCytomation, Denmark) for VEGFR-1 and VEGFR-2], using specific primary antibodies raised against VEGF-A (epitope specific rabbit antibody; Neomarkers, Freemont, CA) diluted 1:100, Flt-1/VEGFR-1 (rabbit polyclonal antibody, clone Ab-1; Neomarkers) diluted 1:50, Flk-1/KDR/VEGFR2 (rabbit polyclonal antibody, clone Ab-1; Neomarkers) diluted 1:50 and CD31/PECAM-1 (rabbit monoclonal antibody, clone Ab-1/ JC/70A; Neomarkers) diluted 1:50. Briefly, deparaffinized and rehydrated sections were immersed in $0.01 \mathrm{M}$ citrate buffer ( $\mathrm{pH} \mathrm{6.0)}$ and microwaved at $700 \mathrm{w}$ for $15 \mathrm{~min}$ for VEGFR-1 and VEGFR-2 antibodies. For VEGF-A and CD31 antibodies, deparaffinized and rehydrated sections were immersed in EDTA buffer (1X, pH 8.0) and placed in a waterbath for $20 \mathrm{~min}$ at $98^{\circ} \mathrm{C}$. Slides were then incubated with $3 \%$ hydrogen peroxide in methanol for $10 \mathrm{~min}$. For VEGF-A and CD31, slides were incubated in Ultravision block solution (Neomarkers) for $10 \mathrm{~min}$ at room temperature before 1-h incubation with the primary antibody. For VEGFR-1 and VEGFR-2, slides were incubated directly with the primary antibody overnight at $4^{\circ} \mathrm{C}$. Then, sections were sequentially washed in $1 \mathrm{X}$ PBS with $0.02 \%$ Tween-20 incubated with biotinylated goat antipolyvalent antibody for $10 \mathrm{~min}$, streptavidin peroxidase for $10 \mathrm{~min}$, and developed with 3,3-diaminobenzidine for $10 \mathrm{~min}$. Slides were counterstained with Mayer's hematoxylin (Merck, Dermstadt, Germany). Negative controls were carried out by omitting the primary antibody; for positive controls, we used normal tonsil tissue for VEGF-A antibody, invasive ductal breast carcinoma for CD31 antibody, and angiosarcoma for VEGFR-1 and VEGFR-2 antibodies, leading to satisfactory immunostaining of the blood vessels.

Assessment of positive reactions in endothelial cells. Microvessel was defined as a single endothelial cell or a cluster of endothelial cells positive for CD31, surrounding a visible lumen clearly separate from adjacent microvessels and from other connective tissue components (17). Likewise, microvascular density (MVD) was also assessed by staining of VEGF-A, VEGFR-1 and VEGFR-2.

Analysis was performed at x200 (x20 objective lens and $\mathrm{x} 10$ ocular lens) magnification, as described in a previous study with slight modifications (18). Microvessels were counted without image analyzer. A median of 10 hot-spot fields was defined as MVD.

Assessment of positive reactions in epithelial cells. VEGF-A, VEGFR-1, and VEGFR-2 expression in epithelial glandular cells was evaluated semi-quantitatively. The positivity index was obtained by counting all epithelial cells in breast structures and by calculating the percentage of cells with VEGF cytoplasmic immunoreactivity, regardless of staining intensity.

VEGF-A, VEGFR-1, and VEGFR-2 expression was determined by two of the authors (ALF and FCS) independently and inconsistencies were resolved by a simultaneous review. 


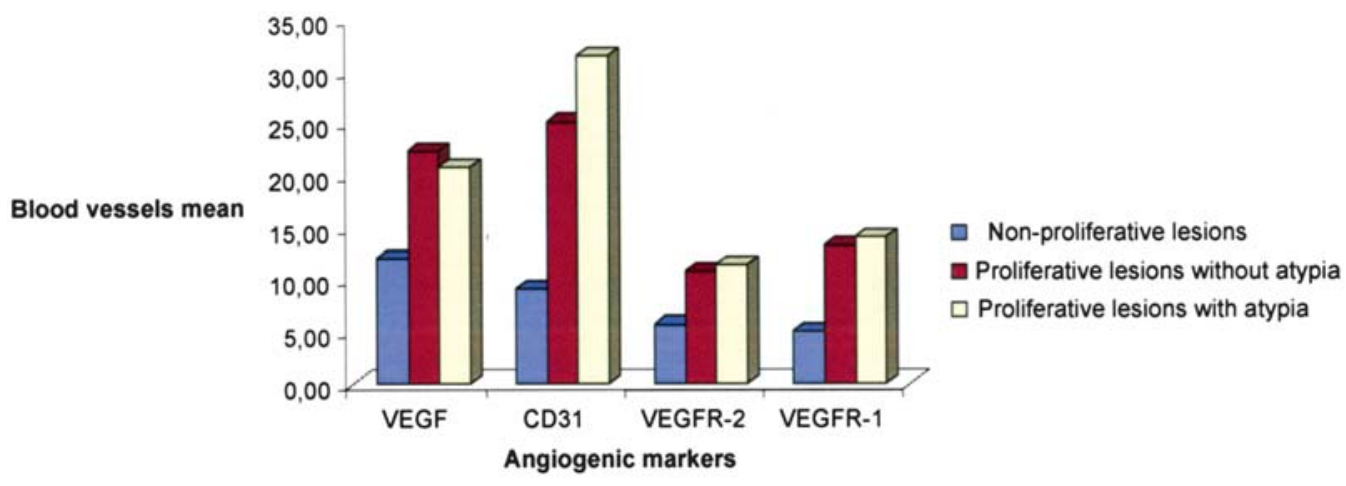

Figure 1. The histogram shows the blood vessel means as assessed by the four angiogenic markers in the categories of breast lesions.

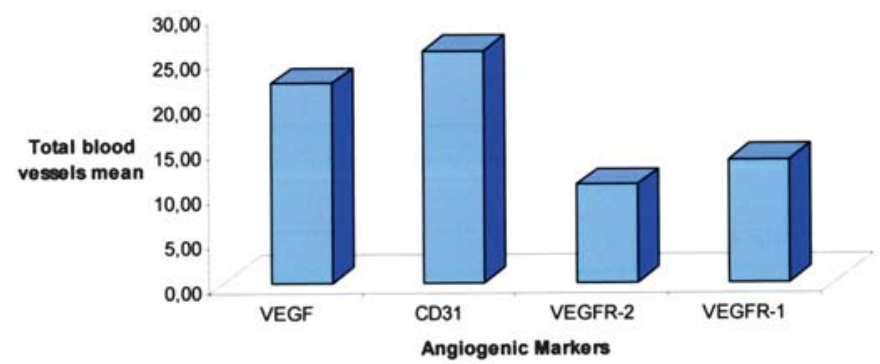

Figure 2. The histogram exhibits the distribution of the four angiogenic markers related to the blood vessel means.

Dynamic angiothermography (DATG) procedure. The DATG examination can be briefly summarized by the following steps (15). i) A very thin layer of plastic coated with black paint and a tiny layer of cholesteric liquid crystals is necessary, the entire structure being held stretched in place by a rigid frame measuring approximately $18 \times 24 \mathrm{~cm}$ in diameter. ii) The plate is placed against the side and front of the breast consecutively and bilaterally, and an incorporated fan cools the skin surface being examined as preferred. iii) The molecular arrangement of the liquid crystals aligns the position along their axis at an angle proportional to the heat picked up from the skin through the fabric, thereby refracting the ambient light in a spectrum of colours ranging from red to violet. It is this physical phenomenon that forms the visible image on the plate and in part matches the underlying arterovenous heat flow. iv) The pattern's rapid appearance and disappearance as the plate is placed against and lifted from the skin yields a functional image in vivo, and hence it is not a measurement of heat as in conventional thermography.

DATG interpretation. DATG allows us to identify the blood supply as flow-lines branching out centripetally from their

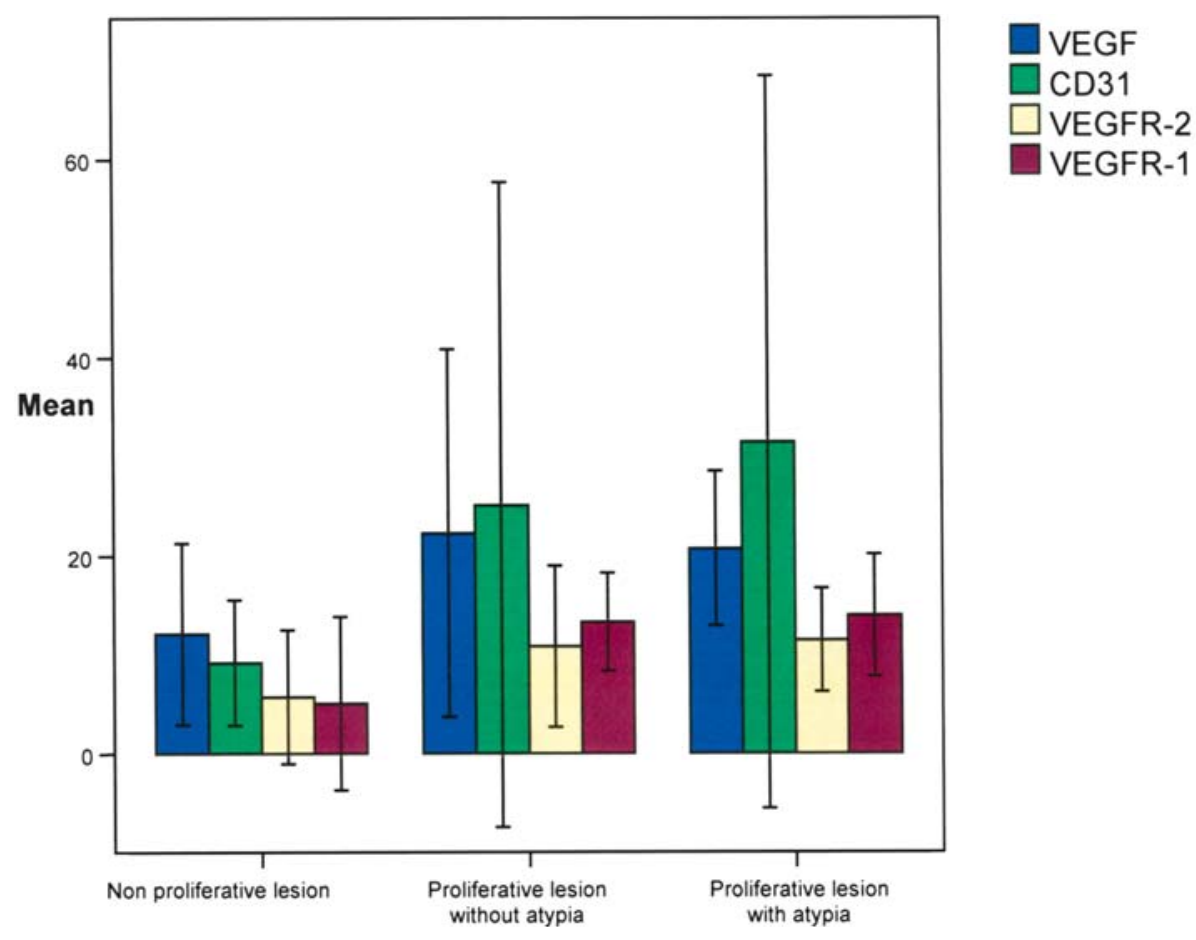

Figure 3. The histogram shows the blood vessel means as assessed by the four angiogenic markers in the categories of breast lesions including the intervals of standard deviation calculated for each marker in each group of lesions. 
Table I. The blood vessel mean and standard deviation values are distributed by the four angiogenic markers in each category of breast lesions.

\begin{tabular}{|c|c|c|c|c|c|}
\hline \multirow[t]{2}{*}{ Diagnostic } & & \multicolumn{4}{|c|}{ Angiogenic markers } \\
\hline & & CD31 & VEGF & VEGFR-2 & VEGFR-1 \\
\hline \multirow[t]{2}{*}{ Non-proliferative lesion } & Mean & 9,200 & 12,133 & 5,73 & 5,07 \\
\hline & Std. Deviation & 6,3411 & 9,1768 & 6,769 & 8,776 \\
\hline \multirow[t]{2}{*}{ Proliferative lesion without atypia } & Mean & 25,142 & 22,292 & 10,85 & 13,33 \\
\hline & Std. Deviation & 32,5925 & 18,5829 & 8,165 & 4,946 \\
\hline \multirow[t]{2}{*}{ Proliferative lesion with atypia } & Mean & 31,460 & 20,740 & 11,48 & 14,00 \\
\hline & Std. Deviation & 36,9987 & 7,8052 & 5,228 & 6,167 \\
\hline P-value & & 0.665 & 0.565 & 0.456 & 0.193 \\
\hline
\end{tabular}

${ }^{a}$ Kruskal-Wallis non-parametric test. Std. Deviation, standard deviation. Correlation between VEGFR-1 and VEGFR-2 expression, $\mathrm{p}<0.0001$.

origin in each plexus in the direction of the nipple and becoming ever thinner until disappearing in a pointed image. This is because the projection of the circulatory heat towards the skin gradually reduces as the branching persists into smaller and smaller vessels and the plate can no longer distinguish the heat from the micro-circulation. The criterion DATG pattern appears on the plate's black surface as a series of sharply pointed triangles, each with its base at the plexus of origin and the apex pointing towards the nipple. DATG rapidly captures any change in blood supply to the whole breast, or any particular part consequent to a physiological or pathological occurrence. Local blood accumulation by a few lobules (even sometimes by a single lobule), for example, clearly appears as evident abnormal flow-lines because they are not pointed at the ends, are not centripetal, are out of proportion with respect to the counter-laterals, or because they extend beyond their territory of origin to another quadrant of the breast. The characteristics and number of these abnormal flow-lines are indicators of malignancy; their absence indicates a likely benign condition.

Biopsies. In the case of non-palpable lesions, an incision was made along the breast's cutaneous tension lines as per the mammogram's point of localization or the anomalous termination of the DATG-detected flow-lines. In the latter case, the exact point was marked with a pencil on the seated patient and $1 \mathrm{cc}$ of methylene blue was injected into the spot immediately before surgery. The tissue for histological analysis was then removed perpendicularly from the skin to the pectoral muscle in a single sample $3-4 \mathrm{~cm}$ wide. Remodeling of the affected area enabled good reconstruction with goodto-optimum aesthetic results. Stereotaxis was not employed for DATG localization as its pattern is solely two-dimensional and, hence, the lesions are not visible.

\section{Results}

The results of positive immunoreactions for CD31, VEGF, VEGFR-1 and VEGFR-2 showed a clear predominance in the breast lesions where cellular atypia was found. The expression of CD31 and VEGF was strongly observed in the lesions categorized as proliferative lesions, with or without atypia, in comparison to non-proliferative lesions. Figs. 1, 2 and 3 illustrate the expression of the angiogenic markers in the three categories of breast lesion.

The histograms in Fig. 1 show the blood vessel means as assessed by the four angiogenic markers in the different categories of breast lesions. CD31 was clearly more expressed in proliferative lesions with atypia when compared with the other markers while VEGF, VEGFR-2 and VEGFR-1 were all similarly expressed in both proliferative lesions with and without atypia.

The histograms of Fig. 2 show the distribution of the four angiogenic markers related to the blood vessel means. CD31 was the marker more importantly expressed by blood vessels (BD), as it had a BD mean slightly higher than that found with VEGF.

The histograms of Fig. 3 demonstrate the intervals of standard deviation (STD) for each marker used in each group of lesions. The STD interval for both markers was very high. The STD values are depicted in Table I according to the angiogenic marker (and its corresponding mean value of blood vessel density) and type of breast lesion. There was a significant correlation between VEGFR-1 and VEGFR-2 expression in all categories of breast lesions $(\mathrm{p}<0.0001)$.

VEGF, VEGFR-1 and VEGFR-2 were also present in epithelial ductal cells with and without atypia. The positive cytoplasmic staining was generally strong and covered large areas of epithelial cells.

In Fig. 4 we can observe blood vessels stained by CD31 surrounding a ductal structure with epithelial proliferation, which was detected by DATG. Epithelial cells also expressed VEGF-A, VEGFR-1 and VEGFR-2. In Fig. 5 this type of positive stain is exemplified by VEGF-A.

Fig. 6 illustrates a case of normal angiothermographic flow-lines. The anatomy is reproduced and the circulation of the acromial plexus of the right breast is exhibited. For comparison with Fig. 6, an abnormal angiothermographic 


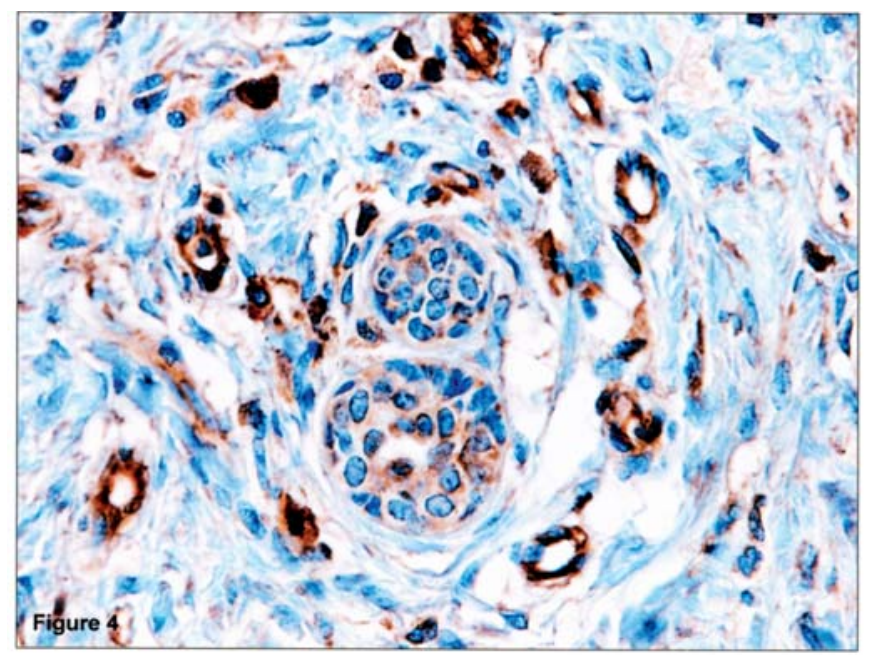

Figure 4. Blood vessels stained by CD31 surrounding a ductal structure with epithelial proliferation, detected by DATG.

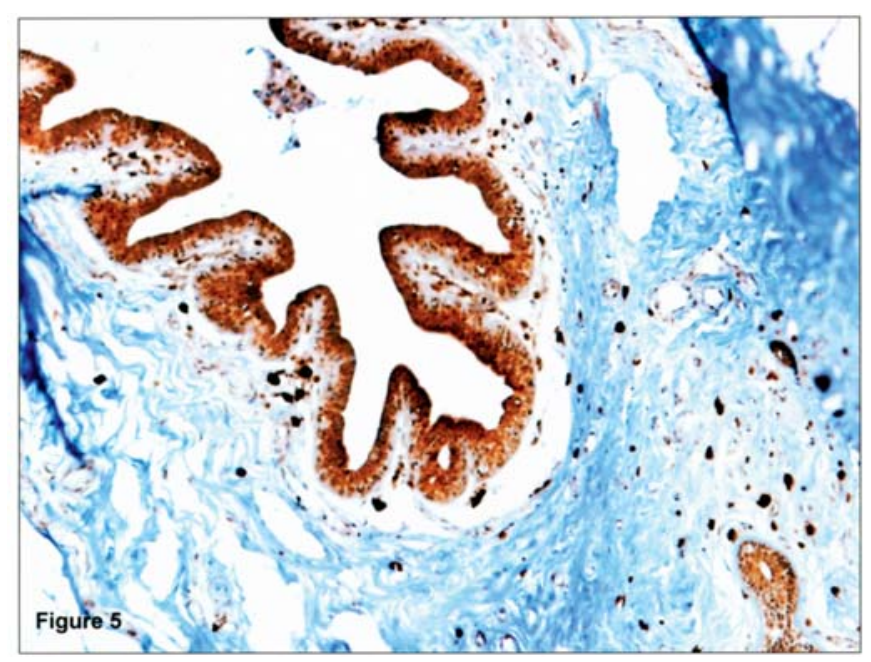

Figure 5. Epithelial cells expressed VEGF-A (x100).

flow-line is shown in Fig. 7. In this example, an upper internal quadrant of the left breast shows a marked anomalous flow caused by a ductal carcinoma in situ.

\section{Discussion}

Expression of the VEGF/VEGFR family seems to extend far beyond the previous expectations of the angiogenic and permeability-inducing factor properties. A wide variety of VEGF family proteins were identified as playing critical functions in lymphangiogenesis, embryonic angiogenesis, inflammation, neuroprotection activity, liver protection and mobilization of bone-marrow derived stem cells. VEGF ligands and receptors are also involved in a plethora of pathological conditions, including cancer and its precursors (10).

VEGF, the most potent angiogenic growth factor, is consistently expressed in all types of pre-invasive lesions of the human breast. The microvascular density (MVD) obtained with immunohistochemical expression of VEGF was higher

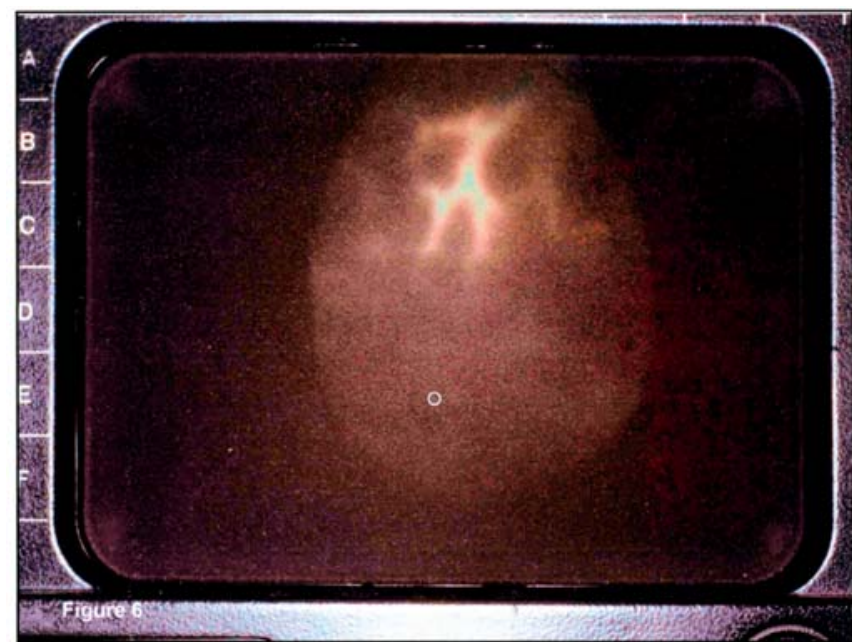

Figure 6. Normal angiothermographic flow-lines. This case reproduces the anatomy of the circulation of the acromial plexus of the right breast. The flow-lines of each plexus should be centripetal, fade out as they terminate in their own area and be proportional to the contralateral.

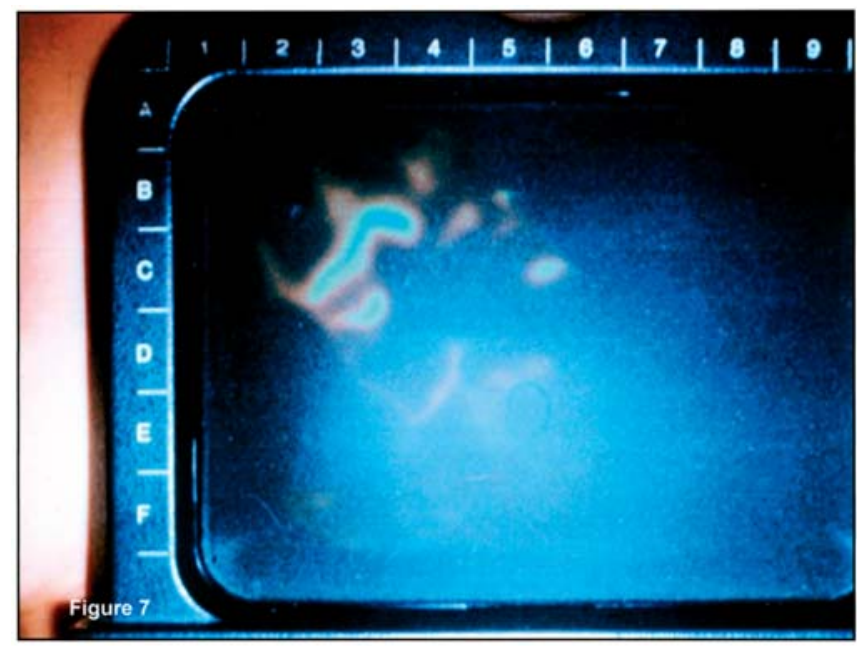

Figure 7. Abnormal angiothermographic flow-lines. Upper internal quadrant of the left breast showing a marked anomalous flow-line formed by countless vessels activated by a ductal carcinoma in situ.

in the lesions with major alterations than in those with minor alterations. It was demonstrated that high MVD is constantly found in breast cancer; conversely, low MVD is more frequently associated with breast structures within the limits of normality (19). These findings are important paradigms because they illustrate that increased vascular density indeed occurs when the cellular metabolic demand is more significantly observed in pathological than in physiological conditions (18).

In the light of our results these premises were not fully confirmed. We observed an increasing expression of MVD from the non-proliferative lesions to proliferative, chiefly in those cases where atypia was observed. At first glance, the histograms in Fig. 1 show that the angiogenic markers are more importantly expressed in proliferative than in nonproliferative lesions. However, when we examine the STD 
values it is clearly demonstrated that the values of the angiogenic markers among the groups of breast lesions are widely variable and overlapping (Fig. 3). We have found high MVD values in several cases of non-proliferative lesions and low MVD values in proliferative breast lesions. Accordingly, the results we observed are not specific enough to support the hypothesis that MVD is a predictive parameter for breast lesions. In spite of the relevant biological significance of MVD in cancer and its related lesions, there are a number of conflicting results regarding the capacity of microvessel quantification in breast carcinomas to predict patient outcome and the existence of metastasis (20).

Concerning the use of dynamic angiothermography, qualitative rather than quantitative image interpretation seems to be an important factor for DATG consistency. Indeed, we found that minimal lesions can trigger functional blood flowlines of many centimeters long; their histopathological features and their corresponding MVD demonstrated a tendency to be higher in atypical than in typical breast lesions. From a clinical point of view, DATG is particularly remarkable because it takes into account that detection of blood flow changes are potentially useful to improve breast lesion diagnosis.

Finally, we found that VEGF was diffusely and strongly expressed in epithelial cells of proliferative lesions in all breast lesion categories studied. VEGFR-1 and VEGFR-2 also stained the epithelial structures but not so intensely as VEGF. This is particularly important because VEGF is a potent blood endothelial vessel growth factor. Its expression in non-cancerous proliferative lesions endorses the crucial role of angiogenesis in sustaining breast lesion development and also ratifies that these lesions can be detected by dynamic angiothermography.

\section{Acknowledgements}

This study was partially supported by grants from FIGO.

\section{References}

1. Cancer Facts and Figures. American Cancer Society, 2004.

2. Cobleigh MA, Vogel CL, Tripathy D, Robert NJ, Scholl S, Fehrenbacher L, Wolter JM, Paton V, Shak S, Lieberman G and Slamon DJ: Multinational study of the efficacy and safety of humanized anti-HER2 monoclonal antibody in women who have HER2-overexpressing metastatic breast cancer that has progressed after chemotherapy for metastatic disease. J Clin Oncol 17: 2639-2642, 1999.
3. Suzuki T, Toi M, Saji S, Horiguchi K, Aruga T, Suzuki E, Horiguchi S, Funata N, Karasawa K and Kamata N: Early breast cancer. Int J Clin Oncol 11: 108-119, 2006.

4. Linderholm B, Grankvist K, Wilking N, Johansson M, Tavelin B and Henriksson R: Correlation of vascular endothelial growth factor content with recurrences, survival, and first relapse site in primary node-positive breast carcinoma after adjuvant treatment. J Clin Oncol 18: 1423-1431, 1998.

5. Wu Y, Hooper AT, Zhong Z, Witte L, Bohlen P, Rafii S and Hicklin DJ: The vascular endothelial growth factor receptor (VEGFR-1) supports growth and survival of human breast carcinoma. Int J Cancer 119: 1519-1529, 2006.

6. Dales JP, Garcia S, Bonnier P, Duffaud F, Carpentier S, Djemli A, Ramuz O, Andrac L, Lavaut M, Allasia C and Charpin C: Prognostic significance of VEGF receptors, VEGFR-1 (Flt-1) and VEGFR-2 (KDR/Flk-1) in breast carcinoma. Ann Pathol 23: 297-305, 2003.

7. Costa C, Soares R and Schmitt F: Angiogenesis: now and then. APMIS 112: 402-412, 2004

8. Meunier-Carpentier S, Dales JP, Djemli A, Garcia S, Bonnier P, Andrac-Meyer L, Lavaut MN, Allasia C and Charpin C: Comparison of the prognosis indication of VEGFR-1 and VEGFR-2 and Tie2 receptor expression in breast carcinoma. Int J Oncol 26: 977-984, 2005.

9. Weis SM and Cheresh DA: Pathophysiological consequences of VEGF-induced vascular permeability. Nature 437: 497-504, 2005.

10. Takahashi $\mathrm{H}$ and Shibuya $\mathrm{M}$ : The vascular endothelial growth factor (VEGF)/VEGF receptor system and its role under physiological and pathological conditions. Clin Sci 109: 227-241, 2005.

11. Wright $\mathrm{T}$ and McGechan A: Breast cancer: new technologies for risk assessment and diagnosis. Mol Diagn 7: 49-55, 2003.

12. Ng EY and Sudharsan NM: Computer simulation in conjunction with medical thermography as an adjunct tool for early detection of breast cancer. BMC Cancer 28: 4-17, 2004.

13. Isard JH, Sweitzer CJ and Edelstien GR: Breast thermography: a prognostic indicator for breast cancer survival. Cancer 62: 484-488, 1988.

14. Folkman J: How is blood vessel growth regulated in normal and neoplastic tissue? G.H.A. Clowes memorial Award lecture. Cancer Res 46: 467-473, 1986.

15. Montruccoli GC, Montruccoli DS and Casali F: Physicochemical characterization of a new plate for breast contact thermography. Physica Medica 20: 27-31, 2004.

16. Tavassoli FA and Deville P: Tumours of the Breast and Female Genital Organs. World Health Organization Classification of Tumours. IARC Press, WHO, Lyon, 2003.

17. Viacava P, Naccarato AG, Bocci G, Fanelli G, Aretini P, Lonobile A, Evangelista G, Montruccoli G and Bevilacqua G: Angiogenesis and VEGF expression in pre-invasive lesions of the human breast. J Pathol 204: 140-146, 2004.

18. Naccarato AG, Viacava P, Bocci G, Fanelli G, Aretini P, Lonobile A, Montruccoli G and Bevilacqua G: Definition of the microvascular pattern of the normal human adult mammary gland. J Anat 203: 599-603, 2003.

19. Ferrara $\mathrm{N}$ and Davis-Smyth T: The biology of vascular endothelial growth factor. Endocr Rev 18: 4-25, 1997.

20. Marinho A, Soares R, Ferro J, Lacerda M and Schmitt FC: Angiogenesis in breast cancer is related to age but not to other prognostic parameters. Pathol Res Pract 193: 267-273, 1997. 